\title{
summary
}

\section{Poor quality studies give insufficient evidence on other possible negative effects of fluoridation}

\author{
McDonagh M, Whiting P, Bradley M, Cooper J, Sutton A, Chestnutt I, et al. A Systematic Review of Public Water
} Fluoridation. York: Publications Office, NHS Centre for Reviews and Dissemination, University of York. ISBN 1900640 16 3; 2000

Objective Does water fluoridation have negative effects? This objective was broken down into four sections: fluorosis, bone fracture and bone development effects, cancer and other possible adverse effects.

Data sources See page 37.

Study selection Thirty-two studies of other possible negative effects were included. These examined various outcomes, including Down's syndrome, mortality, senile dementia, goitre and IQ. All were of evidence level $\mathrm{C}$ with a mean validity score 2.7 (range, 1.5-4.5) out of 8 . Although 22 studies mentioned potential confounding factors, only six used an analysis that controlled for them.

Data extraction and synthesis See page 37.

Results Three of the studies found significant effects. One found a significant negative effect of water fluoride on Alzheimer's disease (increased incidence) and a significant positive effect on impaired mental functioning (decreased incidence). Another found a significant positive association with congenital malformations in one of two sets of data. A third study found that the combination of low iodine and high fluoride concentrations was associated with goitre and learning difficulties. Because of the varying outcome measures the results could not be formally pooled.

Conclusions Interpreting the results of the other possible negative effects is very difficult because of the small number of studies that met inclusion criteria on each specific outcome, because of the study designs used, and also the low quality of the studies. Overall, the studies examining other possible negative effects provide insufficient evidence on any particular outcome to reach conclusions.
Evidence-Based Dentistry
(2002)
3, 49. doi:10.1038/sj. ebd.6400111

\footnotetext{
Address for reprints: Publications Office, NHS Centre for Reviews and Dissemination, University of York, York YO10 5DD, UK. Tel: +44 (0)1904 433648; fax: +44 (0)1904 433661; e-mail: crdpub@york.ac.uk
}

\section{Commentary}

A wide range of negative effects have been attributed to water fluoridation. Fluorosis, cancer and bone fractures were considered in detail in the review. This section of the York review included 33 papers of level $\mathrm{C}$ evidence, the lowest quality of evidence with highest risk of bias. The overall validity scores of the individual studies were also low. Studies on the following were included: Alzheimer's disease, impaired mental functioning, primary degenerative dementia, cognitive impairment, anaemia during pregnancy, age at menarche, congenital malformations, Down's syndrome, infant mortality, Sudden Infant Death Syndrome, mortality, IQ, mental retardation, skeletal fluorosis and goitre.

The review clearly emphasised the relationship between dental fluorosis and water fluoridation but the evidence relating to other negative effects is weak. Although there is more evidence relating to cancer and bone fractures it is of level $\mathrm{C}$ and shows no association. The evidence of other negative effects of fluoridation is insufficient to reach conclusions.
The effects of fluorosis are visible early, however. We do need to remain vigilant for possible adverse effects which may be the result of long-term exposure: there is a need for highquality research using appropriate analytical methods to control for confounding factors.

David Thomas

Thames Valley Dental Public Health Network, Oxford, UK. 